\title{
Investigations of Bending Singularity Orders in a V-notched Composite Laminate Plate Based on the Ressiner-Mindlin Theory
}

\author{
Chung-De Chen \\ Department of Mechanical Engineering, National Cheng Kung University, Tainan 70101, Taiwan
}

\begin{abstract}
In this paper, the bending singularity at the apex of a V-notched composite laminate plate is investigated. The anisotropy of the laminate is modeled by the Stroh formalism. Based on the eigenfunction expansion method, the bending singularity orders can be determined by solving an eigenvalue problem numerically. The singularity orders depend on the plate angle, material orientation, material anisotropy and the laminate stacking sequence. The comparison cases show that the material orientation should avoid in order to reduce the bending singularity. The layers near the free surfaces have more significant effects on the singularity order. The findings presented in this paper are helpful in the design of the composite laminate with V-notch.
\end{abstract}

\section{Introduction}

The V-notch structures are common seen in many engineering applications. High stress concentrations occur near the notch. For sharp notch tip, the stress singularities occur mathematically. The stress singularity at sharp notch tip was first investigated by Williams [1]. He considered a notched plate subjected to inplane loads. $\mathrm{He}$ introduced the eigenfunction expansion method to find the asymptotic stress field near the notch tip. The concept of the stress singularity orders was also introduced by Williams. Theocaris introduced the complex formulation to solve the singularity order in plane elasticity [2]. After these pioneers' efforts, the problems related to stress singularities were extended to anisotropic materials [3], muli-material wedges [4] and functional materails [5].

For bending singularities in notched plate subjected out-of-plane bending, Williams was also the first researcher to investigate such problem [6]. His results were based on the classical plate theory. However, Hartranft and Sih proved that the classical plate theory lead to an incorrect result of singular stresses [7]. They found that for cracked problem, the first order shear deformation theory (Ressiner-Midlin plate theory) would be a better alternative. Huang investigated the bending singularity based on the Ressiner-Mindlin plate theory [8] Recently, Chen investigated the bending and shear singularity of notched plate [9]. In Chen's results, the stress intensities and the singularity orders were solved simultaneously based on the Mellin transform.

For formulations of anisotropic materials, two systems, The Leknitskii formalisms and Stroh formalisms were proposed [10]. These two formulations were also utilized to solve the stress singularity problems in notched structures $[11,12]$. Recently, Chen investigated bending singularities of an anisotropic notched plate [13]. He used the Mellin transform to solve the stress intensities and the results agreed with the finite element calculations.

In the papers regarding to bending stress singularities $[8,9,13]$, the plate is restricted to be homogeneous. To the best of the author's knowledge, no attempt can be found in the open literature for bending singularities in notched laminated plate. In this paper, we extend the homogeneous anisotropic plate to composite laminate plates. The anisotropy of the laminate is modeled by the Stroh formalism. Based on the eigenfunction expansion method, the bending singularity is investigated.

This paper is organized as follows. In the Introduction section, a literature review regarding to the stress singularities is presented. The motivation of this study is also given in this chapter. The second chapter gives a brief review of the Ressiner-Mindlin composite laminate. The governing equations in terms of displacement and rotations are derived in detail. In the third chapter, a laminate plate with sharp notch is considered. The Stroh formalism is used to model the anisotropy of the material. The eigenfunction expansion method is introduced to determine the bending singularity. In the chapter of Results and Discussions, comparison cases are considered and the numerical results are presented. Discussions on the strength of bending singularity orders for various conditions are presented in this chapter. Finally, concluding remarks are given in the last chapter.

\section{Formulations of ressiner-mindlin composite laminate}


Consider a Ressiner-Mindlin laminate shown in Fig. 1. The displacements in rectangular coordinate

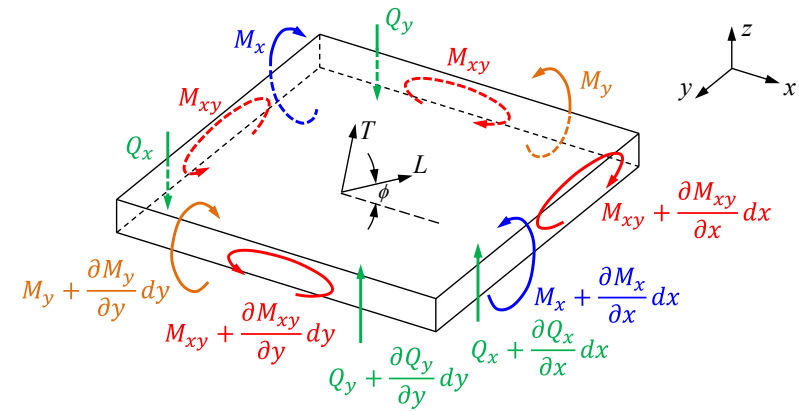

(a)

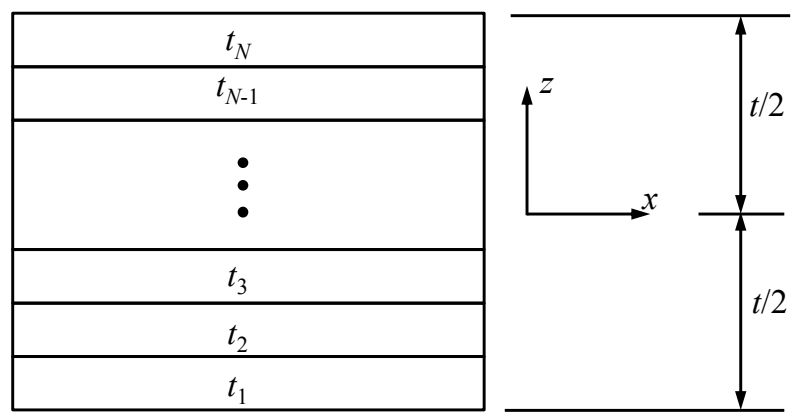

(b)

Figure 1. A Ressiner-Mindlin anisotropic laminate: (a) laminate subjected to moments and shear forces; (b) laminate stacking order.

$$
\begin{aligned}
u_{x}(x, y, z) & =u_{x 0}(x, y)-z \psi_{x}(x, y) \\
u_{y}(x, y, z) & =u_{y 0}(x, y)-z \psi_{y}(x, y) \\
w & =w(x, y)
\end{aligned}
$$

According to the strain-displacement relation, the strains in rectangular coordinate

$$
\begin{gathered}
\varepsilon_{x}=-z \frac{\partial \psi_{x}}{\partial x}, \varepsilon_{y}=-z \frac{\partial \psi_{y}}{\partial y}, \varepsilon_{z}=0 \\
\gamma_{x y}=-z\left(\frac{\partial \psi_{x}}{\partial y}+\frac{\partial \psi_{y}}{\partial x}\right), \gamma_{x z}=\frac{\partial w}{\partial x}-\psi_{x}, \gamma_{y z}=\frac{\partial w}{\partial y}-\psi_{y}
\end{gathered}
$$

The constitutive relation is

$$
\left\{\begin{array}{l}
\sigma_{x} \\
\sigma_{y} \\
\tau_{y z} \\
\tau_{x z} \\
\tau_{x y}
\end{array}\right\}=\left\{\begin{array}{c}
-z\left(Q_{11} \frac{\partial \psi_{x}}{\partial x}+Q_{12} \frac{\partial \psi_{y}}{\partial y}+Q_{16}\left(\frac{\partial \psi_{x}}{\partial y}+\frac{\partial \psi_{y}}{\partial x}\right)\right) \\
-z\left(Q_{12} \frac{\partial \psi_{x}}{\partial x}+Q_{12} \frac{\partial \psi_{y}}{\partial y}+Q_{26}\left(\frac{\partial \psi_{x}}{\partial y}+\frac{\partial \psi_{y}}{\partial x}\right)\right) \\
Q_{44}\left(\frac{\partial w}{\partial y}-\psi_{y}\right)+Q_{45}\left(\frac{\partial w}{\partial x}-\psi_{x}\right) \\
Q_{45}\left(\frac{\partial w}{\partial y}-\psi_{y}\right)+Q_{55}\left(\frac{\partial w}{\partial x}-\psi_{x}\right) \\
-z\left(Q_{16} \frac{\partial \psi_{x}}{\partial x}+Q_{26} \frac{\partial \psi_{y}}{\partial y}+Q_{66}\left(\frac{\partial \psi_{x}}{\partial y}+\frac{\partial \psi_{y}}{\partial x}\right)\right)
\end{array}\right\}
$$

where $Q_{i j}$ denotes the reduced elastic constants, which is given by

$$
Q_{i j}=\left\{\begin{array}{l}
C_{i j}-\frac{C_{i 3} C_{3 j}}{C_{33}}, i j=11,22,12,16,26,66 \\
C_{i j}, i j=44,55,45
\end{array}\right.
$$

Here $C_{i j}$ denotes the elastic constant. For $N$-layer laminate, the moments and shear forces are defined as

$$
\begin{gathered}
M_{x}=-\sum_{k=1}^{N} \int_{h_{k-1}-t / 2}^{h_{k}-t / 2} z \sigma_{x}^{(k)} d z, M_{y}=-\sum_{k=1}^{N} \int_{h_{k-1}-t / 2}^{h_{k}-t / 2} z \sigma_{y}^{(k)} d z, \\
M_{x y}=-\sum_{k=1}^{N} \int_{h_{k-1}-t / 2}^{h_{k}-t / 2} z \tau_{x y}^{(k)} d z \\
Q_{x}=\sum_{k=1}^{N} \int_{h_{k-1}-t / 2}^{h_{k}-t / 2} \tau_{x z}^{(k)} d z, Q_{y}=\sum_{k=1}^{N} \int_{h_{k-1}-t / 2}^{h_{k}-t / 2} \tau_{y z}^{(k)} d z
\end{gathered}
$$

where $h$ is the thickness of the plate and

$$
h_{k}=\left\{\begin{array}{l}
0, k=0 \\
\sum_{j=1}^{k} t_{j}, k \neq 0
\end{array}\right.
$$

The stacking order is shown in Figure 1(b). Substitution of Eq. (5) into (3) gives

$$
\begin{gathered}
\left\{\begin{array}{l}
M_{x} \\
M_{y} \\
M_{x y}
\end{array}\right\}=\left[\begin{array}{lll}
D_{11} & D_{12} & D_{16} \\
D_{12} & D_{22} & D_{26} \\
D_{16} & D_{26} & D_{66}
\end{array}\right]\left\{\begin{array}{c}
\partial \psi_{x} / \partial x \\
\partial \psi_{y} / \partial y \\
\partial \psi_{x} / \partial y+\partial \psi_{y} / \partial x
\end{array}\right\} \\
\left\{\begin{array}{l}
Q_{y} \\
Q_{x}
\end{array}\right\}=\left[\begin{array}{cc}
k_{4}^{2} F_{44} & k_{4} k_{5} F_{45} \\
k_{4} k_{5} F_{45} & k_{5}^{2} F_{55}
\end{array}\right]\left\{\begin{array}{l}
\frac{\partial w}{\partial y}-\psi_{y} \\
\frac{\partial w}{\partial x}-\psi_{x}
\end{array}\right\}
\end{gathered}
$$

where $k_{4}$ and $k_{5}$ denote the shear corrected factors, and

$$
\begin{gathered}
D_{11}=\frac{1}{3} \sum_{k=1}^{N}\left[\left(h_{k}-\frac{t}{2}\right)^{3}-\left(h_{k-1}-\frac{t}{2}\right)^{3}\right] Q_{11}^{(k)}, \\
D_{12}=\frac{1}{3} \sum_{k=1}^{N}\left[\left(h_{k}-\frac{t}{2}\right)^{3}-\left(h_{k-1}-\frac{t}{2}\right)^{3}\right] Q_{12}^{(k)}, \\
D_{22}=\frac{1}{3} \sum_{k=1}^{N}\left[\left(h_{k}-\frac{t}{2}\right)^{3}-\left(h_{k-1}-\frac{t}{2}\right)^{3}\right] Q_{11}^{(k)}, \\
D_{16}=\frac{1}{3} \sum_{k=1}^{N}\left[\left(h_{k}-\frac{t}{2}\right)^{3}-\left(h_{k-1}-\frac{t}{2}\right)^{3}\right] Q_{16}^{(k)}, \\
D_{26}=\frac{1}{3} \sum_{k=1}^{N}\left[\left(h_{k}-\frac{t}{2}\right)^{3}-\left(h_{k-1}-\frac{t}{2}\right)^{3}\right] Q_{26}^{(k)}, \\
F_{44}=\sum_{k=1}^{N} Q_{44}^{(k)} t_{k}, F_{55}=\sum_{k=1}^{N} Q_{55}^{(k)} t_{k}, F_{45}=\sum_{k=1}^{N} Q_{45}^{(k)} t_{k}
\end{gathered}
$$

The equilibrium equations in terms of moments and shear forces are

$$
\frac{\partial M_{x}}{\partial x}+\frac{\partial M_{x y}}{\partial y}+Q_{x}=0, \frac{\partial M_{x y}}{\partial x}+\frac{\partial M_{y}}{\partial y}+Q_{y}=0,
$$




$$
\frac{\partial Q_{x}}{\partial x}+\frac{\partial Q_{y}}{\partial y}=0
$$

Substitution of Eq. (7) into Eq. (9) yields

$$
\begin{gathered}
D_{11} \frac{\partial^{2} \psi_{x}}{\partial x^{2}}+D_{12} \frac{\partial^{2} \psi_{y}}{\partial x \partial y}+D_{16} \frac{\partial^{2} \psi_{x}}{\partial x \partial y}+D_{16} \frac{\partial^{2} \psi_{y}}{\partial x^{2}} \\
+D_{16} \frac{\partial^{2} \psi_{x}}{\partial x \partial y}+D_{26} \frac{\partial^{2} \psi_{y}}{\partial y^{2}}+D_{66} \frac{\partial^{2} \psi_{x}}{\partial y^{2}}+D_{66} \frac{\partial^{2} \psi_{y}}{\partial x \partial y} \\
+k_{4} k_{5} F_{45}\left(\frac{\partial w}{\partial y}-\psi_{y}\right)+k_{55}^{2} F_{55}\left(\frac{\partial w}{\partial x}-\psi_{x}\right)=0 \\
D_{16} \frac{\partial^{2} \psi_{x}}{\partial x^{2}}+D_{26} \frac{\partial^{2} \psi_{y}}{\partial x \partial y}+D_{66} \frac{\partial^{2} \psi_{x}}{\partial x \partial y}+D_{66} \frac{\partial^{2} \psi_{y}}{\partial x^{2}} \\
+D_{12} \frac{\partial^{2} \psi_{x}}{\partial x \partial y}+D_{22} \frac{\partial^{2} \psi_{y}}{\partial y^{2}}+D_{26} \frac{\partial^{2} \psi_{x}}{\partial y^{2}}+D_{26} \frac{\partial^{2} \psi_{y}}{\partial x \partial y} \\
+k_{44}^{2} F_{44}\left(\frac{\partial w}{\partial y}-\psi_{y}\right)+k_{4} k_{5} F_{45}\left(\frac{\partial w}{\partial x}-\psi_{x}\right)=0 \\
k_{4} k_{5} F_{45}\left(\frac{\partial^{2} w}{\partial y \partial x}-\frac{\partial \psi_{y}}{\partial x}\right)+k_{5}^{2} F_{55}\left(\frac{\partial^{2} w}{\partial x^{2}}-\frac{\partial \psi_{x}}{\partial x}\right) \\
+k_{4}^{2} F_{44}\left(\frac{\partial^{2} w}{\partial y^{2}}-\frac{\partial \psi_{y}}{\partial y}\right)+k_{4} k_{5} F_{45}\left(\frac{\partial^{2} w}{\partial x \partial y}-\frac{\partial \psi_{x}}{\partial y}\right)=0
\end{gathered}
$$

\section{Determination of the bending singularities}

Fig. 2 shows a anisotropic plate with a sharp V-notch. The plate angle is $\alpha$ (notch opening angle is $2 \pi-\alpha$ ). For bending moment singularity, the transverse shear force $\tau_{x z}$ and $\tau_{y z}$ are finite at the corner. It is noted that the bending moments are of the order of $\partial \psi_{x} / \partial x$, and the transverse shear forces are of the order of $\left(\partial w / \partial x-\psi_{x}\right)$. Therefore, for $r \rightarrow 0$, the term $\partial \psi_{x} / \partial x$ approaches infinity, the term $\partial^{2} \psi_{x} / \partial x^{2}$ approaches infinity, and the term $\left(\partial w / \partial x-\psi_{x}\right)$ is finite. It suggests that, for $r \rightarrow 0$, the last two terms regarded to $\left(\partial w / \partial x-\psi_{x}\right)$ and $\left(\partial w / \partial y-\psi_{y}\right)$ in Eqs. (10a) and (10b) can be neglected. Eqs. (10a) and (10b) can be written as

$$
\begin{gathered}
D_{11} \frac{\partial^{2} \psi_{x}}{\partial x^{2}}+D_{12} \frac{\partial^{2} \psi_{y}}{\partial x \partial y}+D_{16} \frac{\partial^{2} \psi_{x}}{\partial x \partial y}+D_{16} \frac{\partial^{2} \psi_{y}}{\partial x^{2}}+D_{16} \frac{\partial^{2} \psi_{x}}{\partial x \partial y} \\
+D_{26} \frac{\partial^{2} \psi_{y}}{\partial y^{2}}+D_{66} \frac{\partial^{2} \psi_{x}}{\partial y^{2}}+D_{66} \frac{\partial^{2} \psi_{y}}{\partial x \partial y}=0
\end{gathered}
$$$$
D_{16} \frac{\partial^{2} \psi_{x}}{\partial x^{2}}+D_{26} \frac{\partial^{2} \psi_{y}}{\partial x \partial y}+D_{66} \frac{\partial^{2} \psi_{x}}{\partial x \partial y}+D_{66} \frac{\partial^{2} \psi_{y}}{\partial x^{2}}
$$$$
+D_{12} \frac{\partial^{2} \psi_{x}}{\partial x \partial y}+D_{22} \frac{\partial^{2} \psi_{y}}{\partial y^{2}}+D_{26} \frac{\partial^{2} \psi_{x}}{\partial y^{2}}+D_{26} \frac{\partial^{2} \psi_{y}}{\partial x \partial y}=0
$$

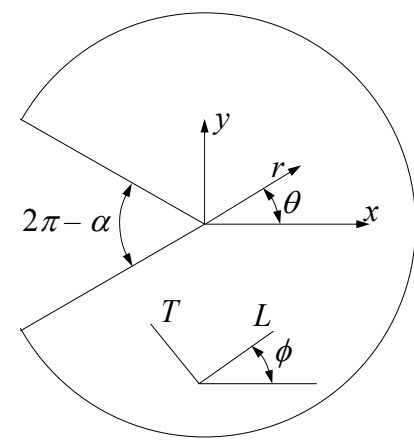

Figure 2. An anisotropic plate with V-notch.

Introducing the complex function $f_{1}$ and $f_{2}$, the rotations and moments can be represented by

$$
\begin{gathered}
\psi_{x}=2 \operatorname{Re}\left[f_{1}^{\prime}+f_{2}^{\prime}\right], \psi_{y}=2 \operatorname{Re}\left[\lambda_{1} f_{1}^{\prime}+\lambda_{2} f_{2}^{\prime}\right] \\
M_{x}=2 \operatorname{Re}\left[\sum_{k=1}^{2}\left(D_{11}+D_{12} \lambda_{k} p_{k}+D_{16}\left(p_{k}+\lambda_{k}\right)\right) f_{k}^{\prime \prime}\right] \\
M_{y}=2 \operatorname{Re}\left[\sum_{k=1}^{2}\left(D_{12}+D_{22} \lambda_{k} p_{k}+D_{26}\left(p_{k}+\lambda_{k}\right)\right) f_{k}^{\prime \prime}\right] \\
M_{x y}=2 \operatorname{Re}\left[\sum_{k=1}^{2}\left(D_{16}+D_{26} \lambda_{k} p_{k}+D_{66}\left(p_{k}+\lambda_{k}\right)\right) f_{k}^{\prime \prime}\right]
\end{gathered}
$$

Where

$$
\begin{aligned}
\lambda_{k} & =-\frac{D_{11}+2 D_{16} p_{k}+D_{66} p_{k}^{2}}{D_{16}+\left(D_{12}+D_{66}\right) p_{k}+D_{26} p_{k}^{2}} \\
& =-\frac{D_{16}+\left(D_{12}+D_{66}\right) p_{k}+D_{26} p_{k}^{2}}{D_{66}+2 D_{26} p_{k}+D_{22} p_{k}^{2}}
\end{aligned}
$$

and $p_{k}$ is the roots of the characteristic equation

$$
\operatorname{det}\left[\mathbf{Q}+p\left(\mathbf{R}+\mathbf{R}^{T}\right)+p^{2} \mathbf{T}\right]=0
$$

Where

$$
\mathbf{Q}=\left[\begin{array}{ll}
D_{11} & D_{16} \\
D_{16} & D_{66}
\end{array}\right], \mathbf{R}=\left[\begin{array}{ll}
D_{16} & D_{12} \\
D_{66} & D_{26}
\end{array}\right], \mathbf{T}=\left[\begin{array}{ll}
D_{66} & D_{26} \\
D_{26} & D_{22}
\end{array}\right]
$$

Assume the complex potentials have the form

$$
f_{k}=a_{k} \zeta_{k}^{-s+2}+b_{k} \zeta_{k}^{-\bar{s}+2}=a_{k} r^{-s+2} \xi_{k}^{-s+2}+b_{k} r^{-\bar{s}+2} \xi_{k}^{-\bar{s}+2}
$$

where $s$ is the eigenvalue and

in which

$$
\begin{aligned}
& \zeta_{k}=x+p_{k} y \\
& \xi_{k}=m+p_{k} n
\end{aligned}
$$




$$
\begin{gathered}
m=\cos \theta \\
n=\sin \theta
\end{gathered}
$$

Substitution of Eq. (18) into Eq. (14) gives

$$
\begin{aligned}
& M_{x}=2 \operatorname{Re}\left[(-s+2)(-s+1) r^{-s} \sum_{k=1}^{2}\left(m_{x}^{(k)} \xi_{k}^{-s} a_{k}+\bar{m}_{x}^{(k)} \bar{\xi}_{k}^{-s} \bar{b}_{k}\right)\right] \\
& M_{y}=2 \operatorname{Re}\left[(-s+2)(-s+1) r^{-s} \sum_{k=1}^{2}\left(m_{y}^{(k)} \xi_{k}^{-s} a_{k}+\bar{m}_{y}^{(k)} \bar{\xi}_{k}^{-s} \bar{b}_{k}\right)\right] \\
& M_{x y}=2 \operatorname{Re}\left[(-s+2)(-s+1) r^{-s} \sum_{k=1}^{2}\left(m_{x y}^{(k)} \xi_{k}^{-s} a_{k}+\bar{m}_{x y}^{(k)} \bar{\xi}_{k}^{-s} \bar{b}_{k}\right)\right]
\end{aligned}
$$

Consider the boundary conditions

$$
\begin{aligned}
& M_{\theta}(\theta=\alpha / 2)=0, M_{\theta}(\theta=-\alpha / 2)=0, \\
& M_{r \theta}(\theta=\alpha / 2)=0, M_{r \theta}(\theta=-\alpha / 2)=0
\end{aligned}
$$

Where

$$
\begin{gathered}
M_{\theta}=n^{2} M_{x}+m^{2} M_{y}-2 m n M_{x y}, \\
M_{r \theta}=-m n M_{x}+m n M_{y}+\left(m^{2}-n^{2}\right) M_{x y}
\end{gathered}
$$

Substitution of Eq. (22) into (23) gives a system of equations in terms of matrix form, i.e.,

$$
\mathbf{M a}=\mathbf{0}
$$

where the $4 \times 4$ matrix $\mathbf{M}$ and $4 \mathrm{x} 1$ vector $\mathbf{a}$ are

$$
\begin{gathered}
\mathbf{a}=\left[\begin{array}{llll}
a_{1} & \overline{b_{1}} & a_{2} & \bar{b}_{2}
\end{array}\right]^{T} \\
\mathbf{M}=\left[\begin{array}{llll}
M_{11} & M_{12} & M_{13} & M_{14} \\
M_{21} & M_{22} & M_{23} & M_{24} \\
M_{31} & M_{32} & M_{33} & M_{34} \\
M_{41} & M_{42} & M_{43} & M_{44}
\end{array}\right] \\
M_{11}=\left.m_{\theta}^{(1)} \xi_{1}^{-s}\right|_{\theta=\alpha / 2}, M_{12}=\left.\bar{m}_{\theta}^{(1)} \bar{\xi}_{1}^{-s}\right|_{\theta=\alpha / 2}, \\
M_{13}=\left.m_{\theta}^{(2)} \xi_{2}^{-s}\right|_{\theta=\alpha / 2}, M_{14}=\left.\bar{m}_{\theta}^{(2)} \bar{\xi}_{2}^{-s}\right|_{\theta=\alpha / 2}, \\
M_{21}=\left.m_{\theta}^{(1)} \xi_{1}^{-s}\right|_{\theta=-\alpha / 2}, M_{22}=\left.\bar{m}_{\theta}^{(1)} \bar{\xi}_{1}^{-s}\right|_{\theta=-\alpha / 2}, \\
M_{23}=\left.m_{\theta}^{(2)} \xi_{2}^{-s}\right|_{\theta=-\alpha / 2}, M_{24}=\left.\bar{m}_{\theta}^{(2)} \bar{\xi}_{2}^{-s}\right|_{\theta=-\alpha / 2} \\
M_{31}=\left.m_{r \theta}^{(1)} \xi_{1}^{-s}\right|_{\theta=\alpha / 2}, M_{32}=\left.\bar{m}_{r \theta}^{(1)} \bar{\xi}_{1}^{-s}\right|_{\theta=\alpha / 2}, \\
M_{33}=\left.m_{r \theta}^{(2)} \xi_{2}^{-s}\right|_{\theta=\alpha / 2}, M_{34}=\left.\bar{m}_{r \theta}^{(2)} \bar{\xi}_{2}^{-s}\right|_{\theta=\alpha / 2}, \\
M_{41}=\left.m_{r \theta}^{(1)} \xi_{1}^{-s}\right|_{\theta=-\alpha / 2}, M_{42}=\left.\bar{m}_{r \theta}^{(1)} \bar{\xi}_{1}^{-s}\right|_{\theta=-\alpha / 2}, \\
M_{43}=\left.m_{r \theta}^{(2)} \xi_{2}^{-s}\right|_{\theta=-\alpha / 2}, M_{44}=\left.\bar{m}_{r \theta}^{(2)} \bar{\xi}_{2}^{-s}\right|_{\theta=-\alpha / 2}
\end{gathered}
$$

For nontrivial solution, we have

$$
\operatorname{det}[\mathbf{M}]=0
$$

The eigenvalue $s$ and the associated bending singularity order $\omega=-S$ can be determined by solving Eq. (29). Mathematically, there are infinity numbers of eigenvalues. Due to the requirement of singularity and the finite value of rotation at the notch tip, only the eigenvalues in the range $0<s<1$ are of interest.

\section{Results and discussions}

In this paper, two materials are considered, the material properties of which are Material A:

$$
\begin{gathered}
E_{L}=10(1+\varepsilon) \mathrm{GPa}, E_{T}=E_{Z}=10 \mathrm{GPa} \\
v_{L T}=v_{L Z}=v_{T Z}=0.25, G_{L T}=G_{L Z}=G_{T Z}=4 \mathrm{Gpa}
\end{gathered}
$$

Material B

$$
\begin{gathered}
E_{L}=132.8 \mathrm{GPa}, E_{T}=E_{Z}=10.76 \mathrm{GPa} \\
G_{L T}=G_{L Z}=5.65 \mathrm{GPa}, G_{T Z}=3.61 \mathrm{GPa}, \\
v_{L T}=0.24, v_{L Z}=v_{L T}, v_{T Z}=0.49
\end{gathered}
$$

In the above material properties, the subscript $L, T$ and $Z$ denote the fiber direction, transverse direction and out-of-plane direction, respectively, $E_{L}, E_{T}$ and $E_{Z}$ are the Young's modulli along $L, T$ and $Z$ directions, respectively, and $v_{L T}, v_{L Z}$ and $v_{T Z}$ are the Poisson's ratios. The laminate is composed of 8layers of lamina. The layer stacking sequence is denoted as $\left[\phi_{1} / \phi_{2} / \phi_{3} / \phi_{4}\right]_{\mathrm{s}}$, where subscript $\mathrm{s}$ denotes the symmetric laminate, $\phi_{1}$ to $\phi_{4}$ denote the angle between $x$-axis and $L$-direction for the first 4 layers. In material A, an anisotropic parameter $\varepsilon$ is introduced. The degree of the anisotropy increses as $\varepsilon$ is large. When $\varepsilon$ is zero, the material degenerates to isotropic.Fig. 3 shows the variations of the bending singularity orders for material A laminate. The stacking sequence is $\left[0^{\circ} / 0^{\circ} / 0^{\circ} / 0^{\circ}\right]_{\mathrm{s}}$. The results reveal that when $\alpha<180^{\circ}$, no singularity is found. Under some plate angle, two eigenvalues are found in the ragne $0<s<1$. Taking the case with very small $\varepsilon\left(\varepsilon=10^{-9}\right)$ as an example, only one eigenvalue exists when $\alpha<258^{\circ}$. When $\alpha>258^{\circ}$, two eigenvalues are found. The results also show that for stronger anisotropy, the singularity order becomes weaker. When plate angle $\alpha=360^{\circ}$, it becomes a cracked plate. The singularity order becomes -0.5 , which coincides with the classical square-root singularity of a crack structure.

Fig. 4 shows the variations of the bending singularity orders for material A laminate with a stacking sequence of $\left[90^{\circ} / 90^{\circ} / 90^{\circ} / 90^{\circ}\right]_{s}$. In this case, the bending singularity is stronger than that shown in Fig. 3 for anisotropic plate. It is noted that for very small $\varepsilon\left(\varepsilon=10^{-}\right.$ ${ }^{9}$ ), the results are identical to those shown in Fig. 3. For example, both the two figures show the same value of $\omega_{1}$ $=-0.4552$. It is also noted that this value agree with the isotropic case appeared in the literature [9]. 


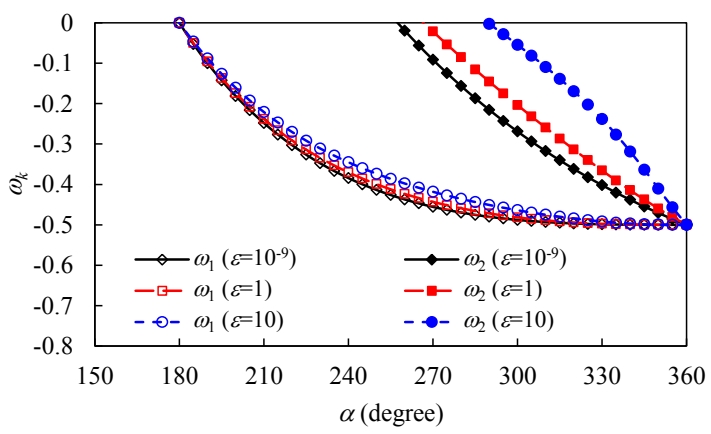

Figure 3. Variations of bending singularity orders for various plate angle $\alpha$. The stacking sequence of the laminate is $\left[0^{\circ} / 0^{\circ} / 0^{\circ} / 0^{\circ}\right]_{\mathrm{s}}$ with mat erial A

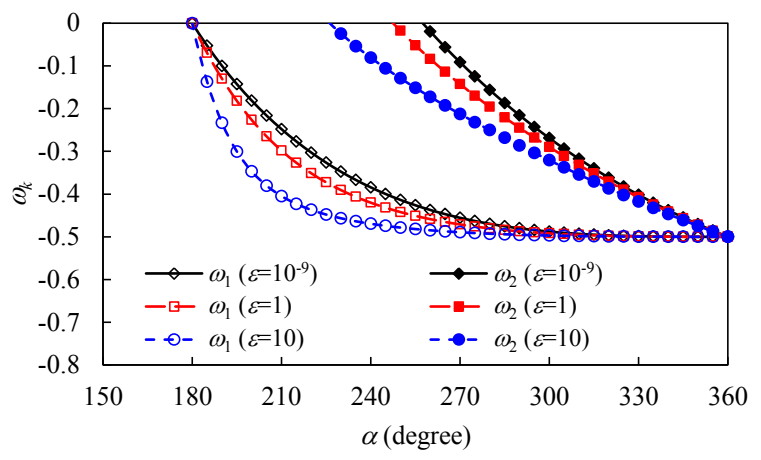

Figure 4. Variations of bending singularity orders for various plate angle $\alpha$. The stacking sequence of the laminate is $\left[90^{\circ} / 90^{\circ} / 90^{\circ} / 90^{\circ}\right]_{\mathrm{s}}$ with material A.

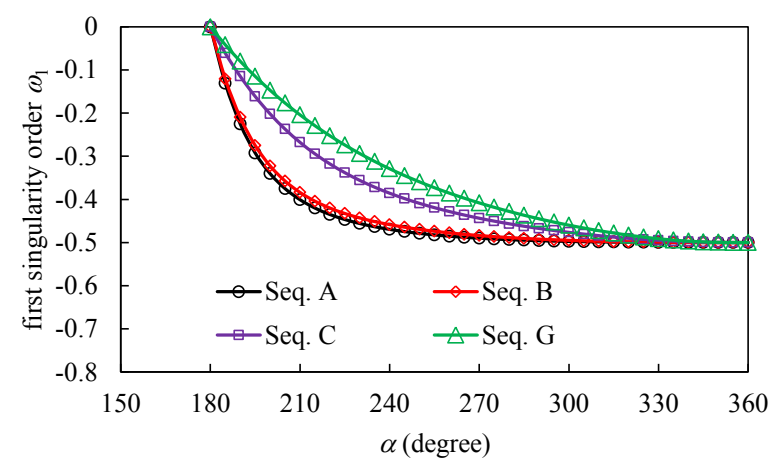

Figure 5. The first bending singularity order for Seq. A, B, C and $\mathrm{G}$ with material $\mathrm{B}$ and for various plate angle $\alpha$.

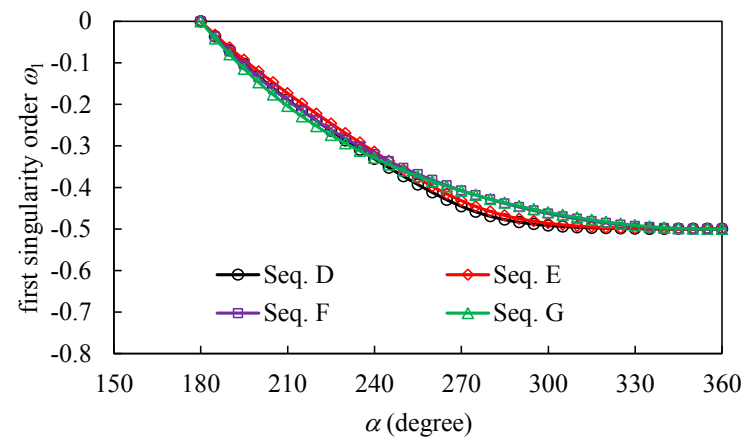

Figure 6. The first bending singularity order for Seq. D, E, $\mathrm{F}$ and $\mathrm{G}$ with material $\mathrm{B}$ and for various plate angle $\alpha$.
Table 1. Stacking sequences

\begin{tabular}{|c|c|}
\hline Seq. & Stacking squence \\
\hline A & {$\left[90^{\circ} / 90^{\circ} / 90^{\circ} / 90^{\circ}\right]_{\mathrm{s}}$} \\
\hline B & {$\left[90^{\circ} / 90^{\circ} / 0^{\circ} / 0^{\circ}\right]_{\mathrm{s}}$} \\
\hline C & {$\left[0^{\circ} / 0^{\circ} / 90^{\circ} / 90^{\circ}\right]_{\mathrm{s}}$} \\
\hline D & {$\left[45^{\circ} / 45^{\circ} / 45^{\circ} / 45^{\circ}\right]_{\mathrm{s}}$} \\
\hline E & {$\left[45^{\circ} / 45^{\circ} / 0^{\circ} / 0^{\circ}\right]_{\mathrm{s}}$} \\
\hline F & {$\left[0^{\circ} / 0^{\circ} / 45^{\circ} / 45^{\circ}\right]_{\mathrm{s}}$} \\
\hline G & {$\left[0^{\circ} / 0^{\circ} / 0^{\circ} / 0^{\circ}\right]_{\mathrm{s}}$} \\
\hline
\end{tabular}

Seven stacking sequences, Seq. A to Seq. G, are considered in this study, as listed in Table 1. Fig. 5 shows the effects of stacking sequence on the bending singularity orders for material B laminate. In this figure, we consider Seq. A, B, C and G. For Seq. A, all the eight layers have the same fiber orientation of $\alpha=90^{\circ}$. For Seq. B, the material orientations of layers 3 to 6 are replaced by $0^{\circ}$. For all considered cases, no singularity is found when $\alpha<180^{\circ}$. It is seen that the first singularity orders for Seq. B are weaker than those of Seq. A. However, the differences are not significant. For Seq. C, the material orientations of first 2 and last 2 layers are replaced by $0^{\circ}$. From the results shown in Fig. 5, it is seen that the singularity orders of Seq. C are significantly weaker than the previous two sequences. From the results shown in Figs. 3 and 4, we see that the singularity orders tend to be stronger when material orientation angle is $90^{\circ}$. For Seq. G, all the eight layers have the same fiber orientation $\left(\alpha=0^{\circ}\right)$. This stacking sequence has the weakest singularity in Fig. 5. For plate bending, the layers near the middle plane have little effect on the plate stiffness. It can be explained that the singularity orders of Seq. B has little difference from those of Seq. A.

Fig. 6 shows the comparisons of the bending singularity for various plate angles. The stacking sequences considered in this figure are Seq. D, E, F and G. Comparing to Seq. A, B, and C, the non-zero fiber orientations are replaced by $45^{\circ}$ in Seq. D, E and F. The numerical results show that no significant difference is observed for the four stacking sequences. The observations from Figs. 5 and 6 suggest that the fiber orientation should avoid $90^{\circ}$ in order to reduce the bending singularity.

\section{Conclusions}

In this paper, the bending singularities near the apex of the V-notch in an anisotropic Ressiner-Mindlin composite laminate are investigated. The constitutive relation is derived based on the lamination theory. The Stroh-like formalism is introduced to model the anisotropy of the material. Based on the eigenfunction expansion method, the bending singularity orders can be determined by solving an eigenvalue problem numerically. The numerical investigations reveal that the bending singularity orders depend on the plate angle, material orientation, material anisotropy and the laminate 
stacking sequence. The material orientation should avoid $90^{\circ}$ in order to reduce the bending singularity, especially for the lamina near the free surface. The findings presented in this paper are helpful in the design of the composite laminate with V-notch.

\section{Acknowledgement}

The author is grateful to the Ministry of Science and Technology of Taiwan for financial support through grant MOST 106-2221-E-006-098-.

\section{References}

1. M.L. Williams: J. Appl. Mech. 19 526-528 (1952)

2. P.S. Theocaris: Int. J. Eng. Sci. 12 107-120 (1974)
3. F. Delale: Int. J. Solids Struct. 20 31-40 (1984)

4. H.P. Chen: Int. J. Solids Struct. 35 1057-1073 (1998)

5. C.H. Chue and C.D. Chen: Int. J. Solids Struct. 39 3131-3158 (2002)

6. M.L. Williams, In: Proceeding of First US National Congress of Applied Mechanics (1952), p. 325-329.

7. R.J. Hartranft and G.C. Sih: J. Math. Phys. 47 37190 (1968)

8. C.S. Huang: Int. J. Mech. Sci. 45 1-20 (2003)

9. C.D. Chen: Appl. Math. Model. 39 7657-7679 (2015)

10. T.C.T. Ting: Anisotropic Elasticity: Theory and Applications (Oxford University Press, New York, 1996).

11. T.C.T. Ting: Int. J. Solids Struct. 17 1057-1068 (1981)

12. C.D. Chen: Int. J. Solids Struct. 43 957-981 (2006)

13. C.D. Chen: Appl. Math. Model. 55 183-204 (2018) 